\title{
Outcomes evaluation after application of multidisciplinary protocol of shared hospital care in patients aged 65 years and older and operated for hip fracture
}

\section{CURRENT STATUS: POSTED}

Research Square

Jorge Salvador Marín

Hospital Universitari Sant Joan d'Alacant

Francisco Javier Ferrández Martínez

Hospital Universitari Sant Joan d'Alacant

José Miguel Seguí Ripoll

Hospital Universitari Sant Joan d'Alacant

José Antonio Quesada Rico

Universidad Miguel Hernandez de Elche

Domingo Orozco Beltrán

Hospital Universitari Sant Joan d'Alacant

Concepcion Carratala-Munuera

Universidad Miguel Hernandez de Elche

maria.carratala@umh.esCorresponding Author

ORCiD: https://orcid.org/0000-0002-1303-6294

José Fernando Martínez López

Hospital Universitari Sant Joan d'Alacant

Juan Carlos Marzo Campos

Universidad Miguel Hernandez de Elche

DOI:

$10.21203 /$ rs.2.13596/v2

SUBJECT AREAS

Geriatrics \& Gerontology

KEYWORDS 
Hip fracture, elderly patient, multidisciplinary shared care protocol, in-hospital mortality, length of hospital stay 


\section{Abstract}

The authors have withdrawn this preprint from Research Square 